\title{
Proyección del Consumo Eléctrico Residencial de la Región Lambayeque Mediante Análisis Univariante Aríma
}

\author{
Projection of the Residential Electricity Consumption of the Lambayeque \\ Region through Univariate Analysis Aríma \\ ${ }^{1}$ Walter Cabrera Torres, ${ }^{2}$ Alexander Cabrera Torres
}

\section{RESUMEN}

Para determinar la proyección del consumo de energía eléctrica residencial de la región Lambayeque, mediante análisis univariante ARIMA, se realizó el siguiente trabajo.

En primer lugar se estabilizó la varianza de la serie que fue estudiada a través de la familia de transformaciones de Box Cox. En segundo lugar se estabilizó la media diferenciando la estructura regular de la serie ya estacionaria en varianza. En tercer lugar se estabilizó la estacionalidad, diferenciándola la estructura estacional de la serie ya estacionaria en media y varianza.

La serie transformada en estacionaria, se le aplicó 11 retardos para obtener las autocorrelaciónes simple y parcial muestral de la estructura regular y 60 retardos para obtener las autocorrelaciónes simple y parcial muestral de la estructura estacional. Estas autocorrelaciones simple y parcial muestral se analizaron con las autocorrelaciones simple y parcial teórica y se obtuvieron las órdenes autorregresivas y medias móviles p,q,P,Q. La estimación de los parámetros o coeficientes $\left(\Theta_{1}\right.$ y $\left.\Theta_{2}\right)$, del modelo ARIMA obtenido, tienen representación estadística.

Palabras clave: Arima, energía eléctrica, proyección y serie estacionaria.

\begin{abstract}
To determine the projection of the residential electric power consumption of the Lambayeque region, by means of ARIMA univariate analysis, the following work was carried out.

First, the variance of the series that was studied through the Box Cox transformation family was stabilized. Second, the mean was stabilized by differentiating the regular structure of the series that was already stationary in variance. In the third place, seasonality was stabilized, differentiating it from the seasonal structure of the already stationary series in mean and variance.

The series transformed into stationary, 11 delays were applied to obtain the simple and partial sample autocorrelations of the regular structure and 60 delays to obtain the simple and partial sample autocorrelations of the seasonal structure. These simple and partial sample autocorrelations were analyzed with the simple and partial theoretical autocorrelations and the autoregressive orders and moving averages $p, q, P, Q$ were obtained. The estimation of the parameters or coefficients $\left(\Theta_{1}\right.$ y $\left.\Theta_{2}\right)$, of the obtained ARIMA model, have statistical representation.
\end{abstract}

Keywords: Arima, electric power, projection and stationary series.

${ }^{1}$ Universidad Nacional de Jaén. Jaén, Cajamarca, Perú.

${ }^{2}$ Universidad Nacional de Frontera. Sullana, Piura, Perú. 


\section{INTRODUCTION}

La electricidad es un bien necesario de consumo masivo que los hogares utilizan como la principal fuente para la iluminación de la vivienda y el funcionamiento del stock de equipos y artefactos eléctricos. Lo cual tiene serias implicancias para el diseño de las políticas públicas y la regulación tarifaria. E1 conocimiento del consumo de energía eléctrica anual en el futuro es necesaria, generalmente, para establecer el calendario de mantenimiento de los sistema de distribución. La información básica para realizar esta predicción fue los datos mensuales de varios años, de la energía demandada. La energía demandada mensualmente se predice en el horizonte temporal de un año. En este período la empresa eléctrica se enfrenta al problema de cubrir una estructura de la demanda cualitativa y cuantitativa potencialmente cambiante que han de satisfacer con una capacidad de distribución que es esencialmente fija o determinada.

A partir de la década de 1970 las enormes incertidumbres que emergieron como consecuencia de la primera crisis del petróleo de 1973, hicieron necesario el desarrollo y aplicación de técnicas mucho más sofisticadas entre las que se encuentran los métodos de usuario final, los métodos econométricos y los métodos Aríma.

La metodología Aríma fue formalizada por Box y Jenkins en 1976, por lo que también se le denomina metodología de Box y Jenkins. Esta metodología Box-Jenkins se diferencia de la mayoría de los métodos porque son los datos los que guían a la especificación del modelo y no la suposición sobre el mismo; utiliza un enfoque iterativo de identificación de un modelo útil a partir de modelos de tipo general.

Esta metodológica consiste en identificar, estimar, validar modelos dinámicos de series y predecir. En la etapa de identificación se puede tener más de un modelo seleccionado; luego en la etapa de estimación de los parámetros del modelo, se verifica a través del estadístico de medición de error, si el modelo es satisfactorio; en la etapa de validación se dice que el modelo se ajusta bien si los residuos entre el modelo de pronóstico y los puntos de datos históricos son reducidos, distribuidos de manera aleatoria e independiente.

La exitosa metodología Arima, por los buenos resultados que se obtienen ha provocado la aplicación a gran cantidad de campos de la economía. En el Perú a partir del año 1996, se desarrolla y se aplican la metodología de Box y Jenkins.

No existió proyecto alguno realizado en la región Lambayeque, que ha utilizado el análisis univariante Arima para la proyección del consumo eléctrico residencial.

En la región Lambayeque, no existe trabajo alguno sobre la proyección del consumo eléctrico residencial, mediante análisis univariante Arima. La metodología de Box y Jenkins o Arima y la demanda de energía, es explicada por los autores como:

Alan Pankratz (1983), mostro claramente como el método Box y Jenkins ó Arima, puede aplicarse a una amplia variedad de conjuntos de datos reales. Wesv, M. \& Harrison, P. J. (1989), presenta un enfoque bayesiano. Andrew C. Harvey (1981,1989), discute procedimientos alternativos para el modelado de series temporales, con especial énfasis hacia la econometría. Peña y Sanchez-Albornoz (1983), presenta una introducción básica de la metodología de Box y Jenkins ó ARIMA, para las ciencias sociales. Brian D.O. Anderson y John B. Moore (1979), se extiende particularmente en sistemas de comunicación y teoría de filtrado digital de señales. Vital para los estudiantes en los campos de control y comunicaciones, su contenido también es relevante a los estudiantes en diversas áreas como la estadística, la economía, la bioingeniería y la investigación. Walter Labys (1999), indica si las predicciones resultan ser demasiado bajas pueden tener lugar a 
carencias de energía cuyos costos habitualmente son mucho mayores que el valor de la energía no suministrada. Por el contario, si las previsiones resultan demasiado altas, los costos de oportunidad pueden ser muy elevados al tener comprometidos, de forma improductiva, cuantiosos fondos económicos durante largos periodos de tiempo. Walter Labys(1999), indica también, que existen al menos tres motivos para modelar la demanda de energía. En primer lugar, el suministro razonablemente fiable de energías es vital para el funcionamiento de la economía moderna. En segundo lugar, la ampliación de los sistemas de suministros de energía requiere muchos años. En tercer lugar, las inversiones necesarias en tales sistemas son altamente intensivas en capital, representando, en algunos países, una considerable proporción de su producto bruto interno. Baltels y Fiebig(1996), afirma que el sector residencial es habitualmente uno de los que principalmente contribuye a las puntas de demanda del sistema de generación eléctrica.

El objetivo del trabajo de investigación fue determinar la proyección del consumo eléctrico Residencial de la Región Lambayeque, mediante el Análisis Univariante ARIMA; se utilizó como único dato el consumo de energía eléctrica mensual, esta data abarco de enero del 2003 a diciembre del 2012 y la proyección fue de enero del 2013 a diciembre del 2014.

\section{MATERIALES Y METODOS}

En el siguiente trabajo de investigación, para el cálculo del modelo ARIMA, se utilizó el SPSSV14. Luego se presentó la secuencia, base para el cálculo de un modelo ARIMA.

\section{Análisis de estabilidad en varianza}

Las series, a lo largo de su evolución histórica pueden presentar una variabilidad no constante, en la mayoría de los casos la variabilidad no aumenta con el tiempo sino con el nivel de la serie. Por tanto, se tendrá que efectuar una transformación de la variable, para estabilizar la varianza.
Prueba de Levene, La prueba de Levene permite comprobar la hipótesis de que los grupos anuales de datos mensuales formados, proceden de poblaciones con varianza común. En el caso de que la hipótesis de homogeneidad de varianzas fuera rechazada, podría ser debido a que la dispersión de la serie cambia con la tendencia central. Una situación frecuente es que en los periodos en los que la tendencia central es grande también lo sea la dispersión. Para estabilizar la varianza se recurre a la familia de transformaciones de Box Cox.

En el siguiente trabajo, para transformar la serie y conseguir un modelo ARIMA adecuado, se aplicó raíz cuadrada según la familia de Box Cox, de acuerdo con el grafico siguiente.

La figura 01, presento la evolución histórica del consumo eléctrico residencial expresado en kilo watt hora (kwh), de la región Lambayeque en el periodo 2003-2012.

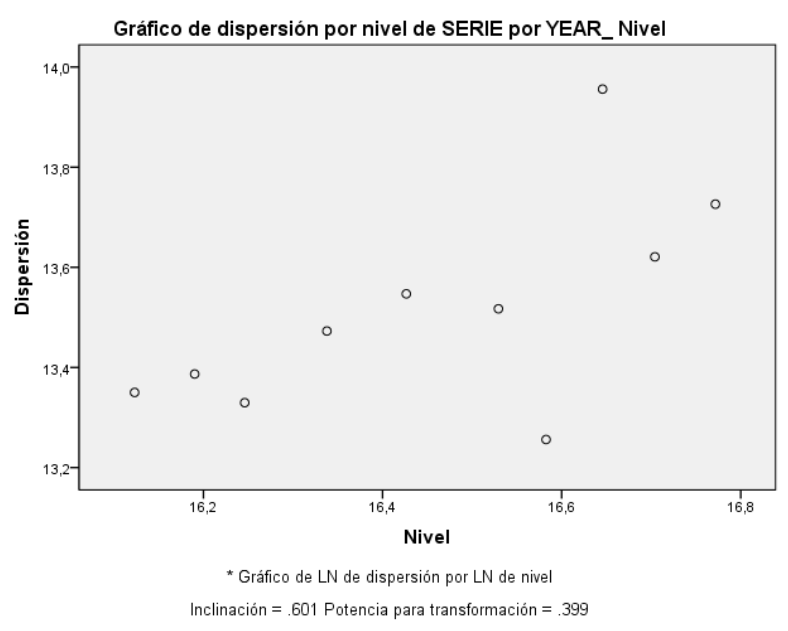

Figura 1. Gráfico de dispersión de la serie del consumo eléctrico residencial de la Región Lambayeque (2003-2012).

\section{Análisis de estabilidad en media y de estacionalidad}

Con la aplicación de diferencias regulares y estacionales, es posible estabilizar la media de la serie. Después de estabilizar la varianza de la serie, se procede a determinar el grado de diferencia, tomando como referencia: 
-El comportamiento gráfico de la serie.

-El comportamiento de las funciones de autocorrelación simple (FAC) y parcial (FACP)

-El uso de estadísticos de medición de error para elegir el mejor arreglo

-El contraste de raíces unitarias para verificar la estacionariedad de la serie.

Si la serie en estudio presenta tendencia creciente o decreciente, su FAC tendrá una estructura positiva con decrecimiento lento hacia cero, entonces esta tendencia puede estabilizarse aplicando sucesivas diferencias regulares:

$$
\nabla^{d} X_{t}=(1-L)^{d} X_{t} \quad \text { Ec. } 1
$$

En el siguiente trabajo de investigación, para estabilizar la media, se diferenció la parte regular $(d=1)$ de la serie en estudio.

Tabla 1. Serie estabilizada en media.

\begin{tabular}{llllll}
\hline \hline \multirow{2}{*}{$\begin{array}{c}\text { Nombre } \\
\text { de serie }\end{array}$} & \multicolumn{2}{c}{$\begin{array}{c}\text { Números de casos de } \\
\text { valores no perdidos }\end{array}$} & $\begin{array}{c}\mathrm{N}^{\circ} \text { de } \\
\text { casos } \\
\text { validos }\end{array}$ & $\begin{array}{c}\text { Creando } \\
\text { función }\end{array}$ \\
\cline { 2 - 3 } & Primero & Último & & \\
\hline 1 & A & 2 & 120 & 119 & DIFF(SQRT,1) \\
\hline \hline
\end{tabular}

La estacionalidad, se manifiesta como una pauta regular de comportamiento periódico en la serie. Si en el gráfico de la serie no se muestra evidente la presencia de estacionalidad, entonces se recurre a representar la FAC. En el caso de que la serie observada presentara estacionalidad de periodo s la FAC mostrará coeficientes altos con decrecimiento lento en los retardos $\mathrm{s}, 2 \mathrm{~s}, 3 \mathrm{~s}, \ldots$.. Entonces la estacionalidad se puede eliminar aplicando diferencias sucesivas estacionales D, de periodos:

$$
\nabla_{s}^{D} X_{t}=\left(1-L^{s}\right)^{D} X_{t} \quad \text { Ec. } 2
$$

En el siguiente trabajo, para estabilizar la estacionalidad, se diferenció la parte estacional $(D=1)$ de la serie en estudio.

Tabla 3. Serie estabilizada en media y estacionalidad.

\begin{tabular}{llllll}
\hline \hline \multirow{2}{*}{$\begin{array}{c}\text { Nombre } \\
\text { de serie }\end{array}$} & \multicolumn{2}{c}{$\begin{array}{c}\text { Números de casos de } \\
\text { valores no perdidos }\end{array}$} & \multirow{2}{*}{$\begin{array}{c}\mathrm{N}^{\circ} \text { de } \\
\text { casos } \\
\text { validos }\end{array}$} & Creando función \\
\cline { 3 - 5 } & Primero & Último & \\
\hline 1 & $\mathrm{C}$ & 14 & 120 & 107 & DIFF(B,1) \\
\hline \hline
\end{tabular}

De las tres series diferenciadas (con nombres " $A$ ", "B" y "C"), se obtiene su media y su desviación típica:

Tabla 4. Estadísticos descriptivos de la serie del consumo eléctrico residencial de la región Lambayeque (2003-20012).

\begin{tabular}{lclc}
\hline \hline & $\mathrm{N}$ & \multicolumn{1}{c}{ Media } & $\begin{array}{c}\text { Desviación. } \\
\text { típica }\end{array}$ \\
\hline DIFF(SQRT,1) & 119 & 10,0175 & 87,67037 \\
SDIFF(SQRT,1,12) & 108 & 133,0301 & 49,44675 \\
DIFF(B,1) & 107 & 0,0345 & 49,23921 \\
N Válidos(según lista) & 107 & & \\
\hline \hline
\end{tabular}

De acuerdo con el cuadro la serie " $C$ " o serie $\operatorname{DIFF}(B, 1)$, que fue diferenciada tanto en la parte regular como estacional, tiene mayor estabilidad en la media por tener el menor valor y también tiene el menor valor de la desviación típica.

3. Determinación de las ordenes p,q de la parte regular y $P, Q$ de la parte estacional.

La identificación de las órdenes autorregresivas y de medias móviles de la parte regular del modelo $(p, q)$, se realiza a partir de la función de autocorrelacion simple (ACF) y función de autocorrelacion parcial (ACFP) muéstrales de la serie que se le aplica en forma simultánea una diferencia regular y una diferencia estacional, las mismas que se comparan con el comportamiento de los retardos típicos de la función de autocorrelacion simple (ACF) y función de autocorrelacion parcial (ACFP) teóricas.

En el siguiente trabajo de investigacion, como un caso de serie temporal; el grafico ACF y ACFP se identificó un orden regular de media móvil q=1, MA(1), cuya característica de la CAF y CAFP es la siguiente. 


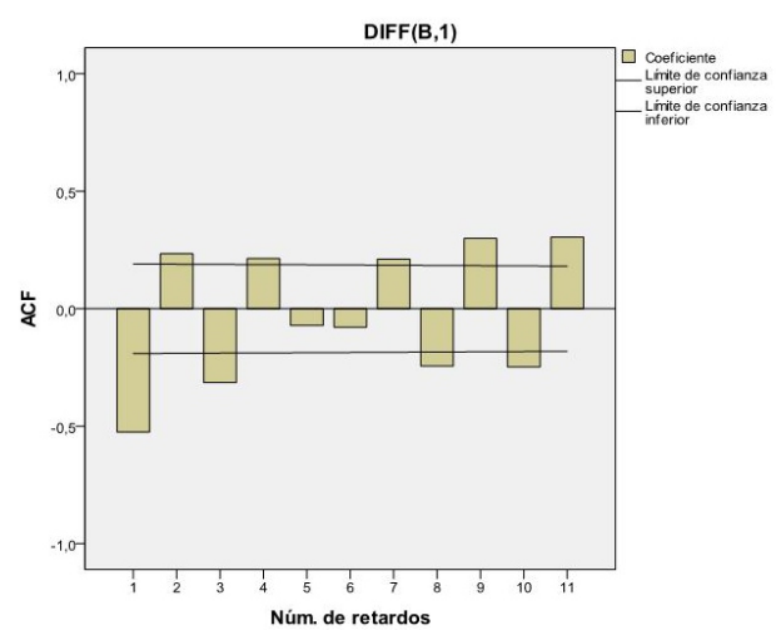

Figura 2. Función de autocorrelacion simple muestral de la estructura regular de la serie en el periodo 2003-2012.

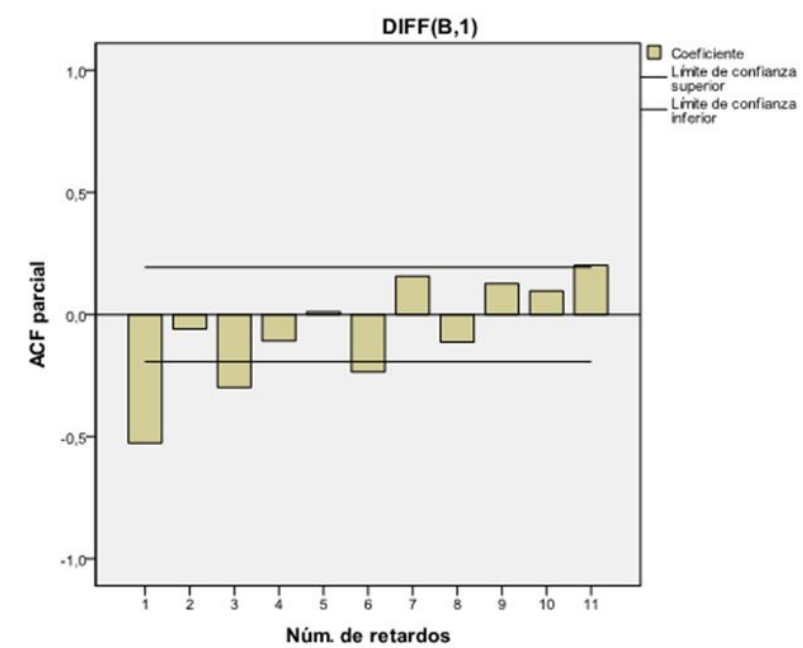

Figura 3. Función de autocorrelacion parcial muestral de la estructura regular de la serie en el periodo 2003-2012.

De la misma manera, la identificación de las ordenes autorregresivos y de medias móviles de la parte estacional (Py Q), se realiza a partir de la función de autocorrelación simple (ACF) y función de autocorrelación parcial (ACFP) muéstrales, de la serie que se le aplica en forma simultánea una diferencia regular y una diferencia estacional, considerando exclusivamente los retardos estacionales $s, 2 \mathrm{~s}$, $3 \mathrm{~s} \ldots . . .$, y teniendo como patrón de comportamiento a las ACF y ACFP teóricas.

En el siguiente trabajo de investigación, como un caso de serie temporal; el gráfico ACFP se identificó un orden estacional de media móvil Q $=1$, MA (1), cuya característica de la ACF y ACFP es la siguiente.

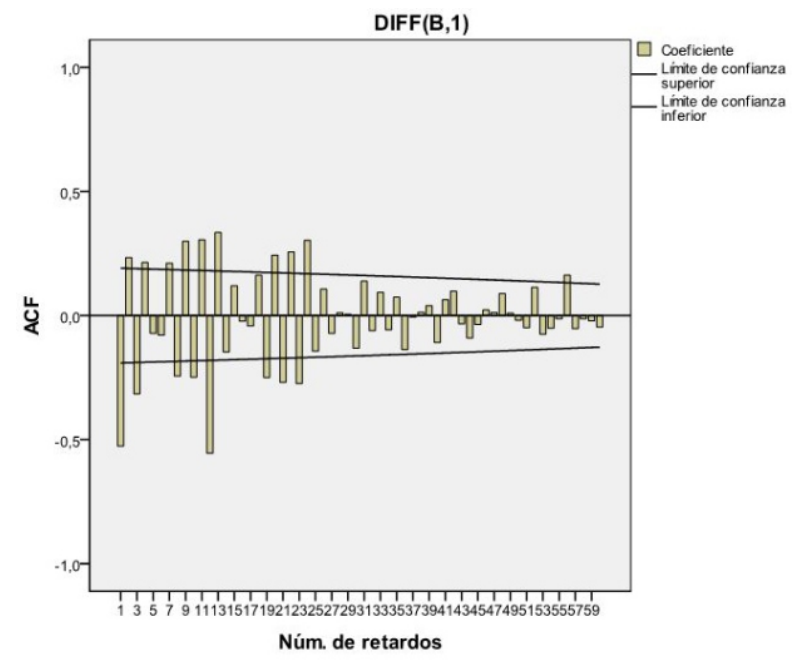

Figura 4. Función de autocorrelacion simple muestral de la estructura estacional de la serie en el periodo 2003-2012.

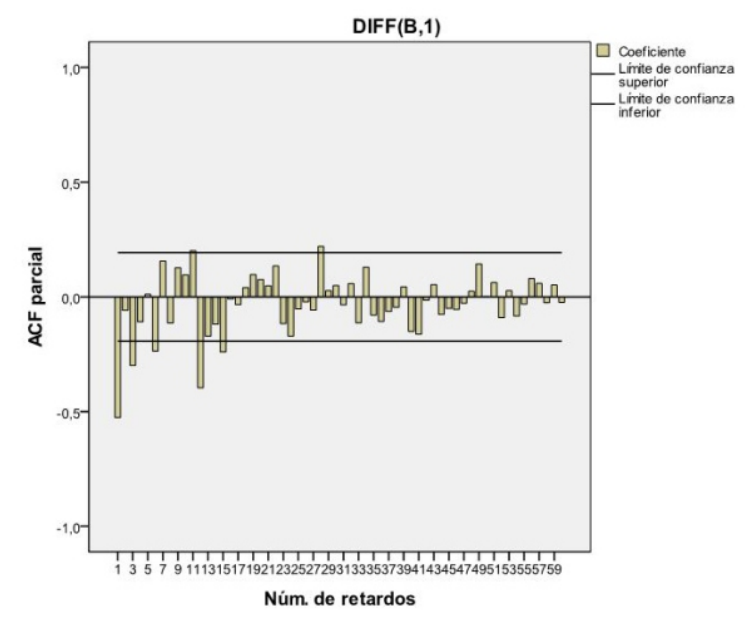

Figura 5. Función de autocorrelacion parcial muestral de la estructura estacional de la serie en el periodo 2003-2012.

\section{Estimación de parámetros}

Una vez determinada las ordenes autoregresivas y medias móviles, se trata de estimar los parámetros o coeficientes del modelo ARIMA $(\varnothing, \Phi, \theta, \Theta)$, cuya expresión general es:

$\Phi_{P}\left(L^{s}\right) \varnothing_{p}(L) \nabla^{d} \nabla_{s}^{D} X_{t}=\theta_{q}(L) \Theta_{Q}\left(L^{s}\right) \partial_{t}+C$

Ec. 3

La estimación de los parámetros $\varnothing, \theta, \Phi$, y $\Theta$ generalmente se realiza por los métodos máxima verosimilitud condicional y máxima verosimilitud exacta. En base a ello es importante 
señalar que los distintos programas que se utilizan, pueden proporcionar valores diferentes de los parámetros calculados para un mismo modelo ARIMA; pues además se suma la diferencia de algoritmos utilizados por cada programa.

En el proceso de ajuste de cada modelo ARIMA tentativo, una vez que se ingresó las ordenes $\operatorname{ARIMA}(\mathrm{p}, \mathrm{d}, \mathrm{q})(\mathrm{P}, \mathrm{D}, \mathrm{Q})$ al programa utilizado, se comprobó si los parámetros calculados por dicho programa son significativamente distintos de cero. Se realizó mediante la probabilidad asociada al estadístico $\mathrm{t}$-Student y (Approx Sig), para contrastar la hipótesis nula de que el parámetro correspondiente es igual a cero.

Para la serie que fue estudiada; se presentó el cuadro de salida en SPSS del modelo ARIMA.

Tabla 5. Parámetros del modelo ARIMA con constante.

\begin{tabular}{|c|c|c|c|c|c|c|}
\hline & & & Estimac & ET & $\mathrm{t}$ & Sig \\
\hline \multirow[t]{5}{*}{$\begin{array}{l}\text { Serie } \\
1\end{array}$} & $\begin{array}{l}\text { Raíz } \\
\text { cuadrada }\end{array}$ & Constante & 0,139 & 0,532 & 0,262 & 0,794 \\
\hline & & Diferencia regular & 1 & & & \\
\hline & & MA regular $\quad$ Retardo 1 & 0,660 & 0,076 & 8,642 & 0,000 \\
\hline & & Diferencia estacional & 1 & & & \\
\hline & & MA estacional $\quad$ Retardo 1 & 0,625 & 0,114 & 5,470 & 0,000 \\
\hline
\end{tabular}

Como

$\theta=0.660 ; \Theta=0.625 ; \mathrm{C}=0.139$

Entonces

$\theta=$ es representativo estadístico.

$\Theta=$ es representativo estadístico.

$\mathrm{C}=$ es no representativo estadístico $(\mathrm{P}>0.05)$

El cuadro de salida en SPSS del modelo ARIMA, presento el valor de los parámetros pero sin la constante del modelo.

Tabla 6. Parámetros del modelo ARIMA sin constante.

\begin{tabular}{|c|c|c|c|c|c|c|}
\hline & & & Estimac & ET & $\mathrm{t}$ & Sig \\
\hline \multirow{3}{*}{$\begin{array}{l}\text { Serie } \\
-1\end{array}$} & \multirow{3}{*}{$\begin{array}{l}\text { Raíz } \\
\text { cuadrada }\end{array}$} & Diferencia regular & 1 & & & \\
\hline & & $\begin{array}{l}\text { MA regular Retardo } 1 \\
\text { Diferencia estacional }\end{array}$ & $\begin{array}{l}0,658 \\
1\end{array}$ & 0,076 & 8,650 & 0,000 \\
\hline & & $\begin{array}{ll}\text { MA } & \text { Retardo 1 } \\
\text { estacional } & \end{array}$ & 0,623 & 0,114 & 5,488 & 0,000 \\
\hline
\end{tabular}

Donde, el parámetro estimado tiene significancia estadística (valor como tal, el calculado), cuando el estadístico t-Student ( $\mathrm{t}$ ) posea un valor no menor de 2 y/o el estadístico Aprox Sig. (p-valor) tenga un valor no mayor de 0.05 .

\section{Proyección del consumo eléctrico residencial.}

Finalmente se procedió a proyectar la serie que fue estudiada. Se tiene un error promedio porcentual histórico de 1.2 y un error promedio porcentual de validación de 1.7.

El gráfico presenta la serie que fue estudiada de color azul y la serie que fue proyectada de color verde.

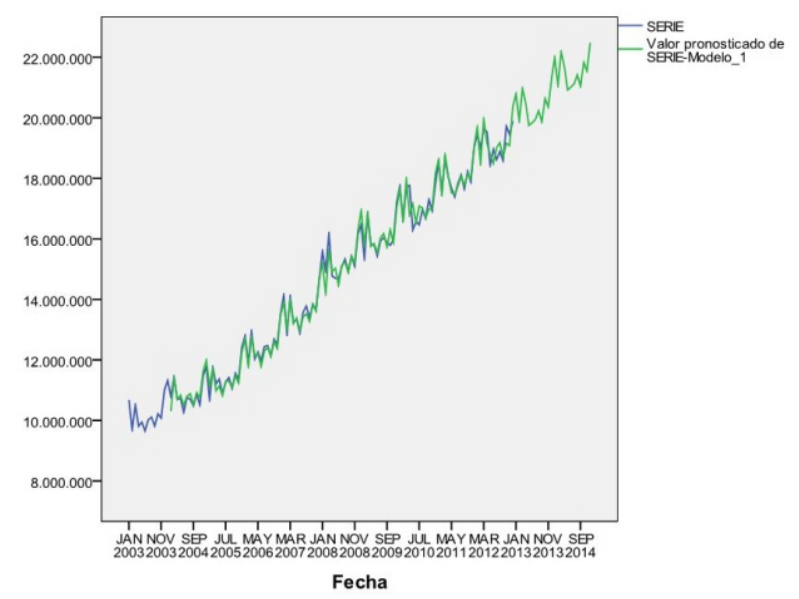

Figura 6. Serie del consumo eléctrico residencial de la región Lambayeque (20032012) VS serie modelada del consumo eléctrico residencial de la Región Lambayeque (20032014).

\section{RESULTADOS}

Con referencia a la figura 1, muestra la estimación de la potencia para transformación $(\lambda)$ de la serie del consumo eléctrico residencial de la Región Lambayeque del periodo 20032012 , cuyo valor fue de $\lambda=0.399$.

Con referencia a la tabla IV, muestra los valores de la media y la desviación típica de la serie diferenciada, previa transformación de la serie a través de la raíz cuadrada (estabilizada en varianza). A la serie estabilizada en varianza se le aplico diferencias de la manera siguiente: 
- Una diferencia regular, cuyo resultado fue una desviación típica de 87,67037 y una media de 10,0175 .

- Una diferencia estacional, cuyo resultado fue una desviación típica de 49,44675 y una media de 133,0301.

- Una diferencia regular y una diferencia estacional, cuyo resultado fue una desviación típica de 49,23921 y una media de 0,0345.

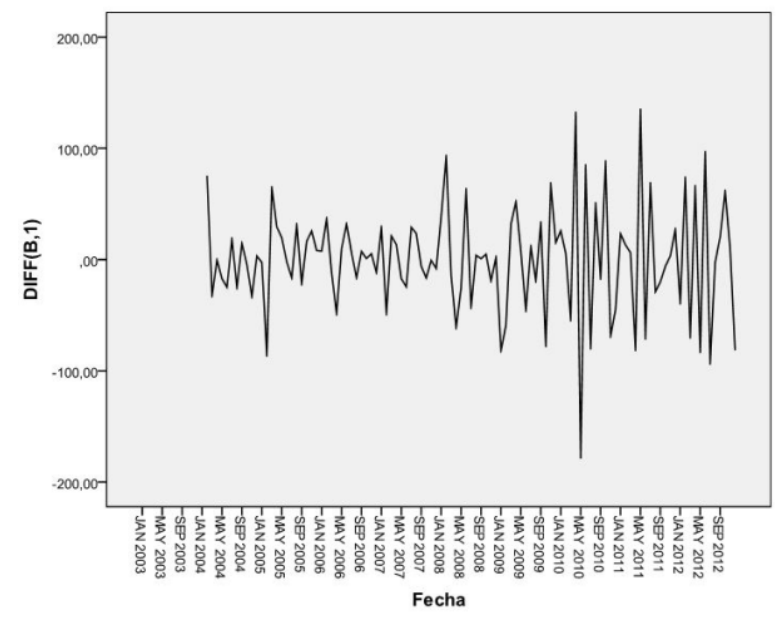

Figura 7. Serie estacionaria del consumo eléctrico residencial de la Región Lambayeque (2003-2012).

En la figura 7, muestra el comportamiento de la serie en el periodo 2003-2012, que fue estabilizada en varianza, media y estacionalidad. Con referencia a la figura 2 y figura 3 , representan la función de autocorrelación simple y parcial muestral de la estructura regular, estas funciones se lograron obtener aplicando 11 retardos a la serie estacionaria del consumo eléctrico residencial de la Región Lambayeque en el periodo 2003-2012. Las funciones de autocorrelación simple y parcial muestral fueron analizados con las funciones de autocorrelación simple y parcial teórica, y se logró obtener la identificación a priori de los siguientes modelos $\operatorname{ARIMA}(p, d, q):(2,1,0),(0,1,2)$ y $(0,1,1)$.

La figura 4 y figura 5 , representan la función de autocorrelación simple y parcial muestral de la estructura estacional, estas funciones se lograron obtener aplicando 60 retardos a la serie en el periodo 2003-2012. Las funciones de autocorrelación simple y parcial muestral fueron analizados con la funciones de autocorrelación simple y parcial teórica, y se logró obtener la identificación a priori de los siguientes modelos ARIMA (P,D,Q): $(1,1,0),(2,1,0),(0,1,2), y$ $(0,1,1)$.

La identificación total de modelos ARIMA $(\mathrm{p}, \mathrm{d}, \mathrm{q})(\mathrm{P}, \mathrm{D}, \mathrm{Q})$ fueron los siguientes: $(2,1,0)(1,1,0),(2,1,0)(2,1,0),(2,1,0)(0,1,2)$, $(2,1,0)(0,1,1),(0,1,2)(1,1,0),(0,1,2)(2,1,0)$, $(0,1,2)(0,1,2),(0,1,2)(0,1,1),(0,1,1)(1,1,0)$, $(0,1,1)(2,1,0),(0,1,1)(0,1,2),(0,1,1)(0,1,1)$

Con referencia a la tabla $\mathrm{V}$, muestra los parámetros estimados con el modelo ARIMA que fue identificado $(0,1,1)(0,1,1)$ y fue el único modelo que cumplió con los parámetros estadísticos determinados.

El estadístico t-student ( $\mathrm{t}$ ), presento valores mayores de 2:

$$
\begin{aligned}
& t=8.642>2 \\
& t=5.470>2
\end{aligned}
$$

Así también el estadístico Aprox Sig (p-valor), sus valores fueron menores de 0.05 , a diferencia de la constante:

$$
\begin{aligned}
& \mathrm{P} \text {-valor }=0.794>0.05 \\
& \mathrm{P} \text {-valor }=0<0.05 ; \mathrm{P} \text {-valor }=0<0.05
\end{aligned}
$$

Entonces

$\theta 1 .-$ tiene representación estadística, cuyo valor es 0.660

$\Theta 1 .-$ tiene representación estadística, cuyo valor es 0.625

$\mathrm{C}$, es no representativo estadístico $(\mathrm{P}>0.05)$, lo que significa estimar nuevamente los parámetros pero sin la constante.

Con referencia a la tabla VI, muestra los nuevos valores de los parámetros que fueron estimados pero sin la constante modelo. La estimación del modelo para la serie del consumo eléctrico residencial de la región Lambayeque fue:

$$
\begin{aligned}
& \Phi_{P}\left(L^{S}\right) \varnothing_{p}(L) \nabla^{d} \nabla_{s}^{D} X_{t}=\theta_{q}(L) \Theta_{Q}\left(L^{S}\right) \partial_{t} \\
& \nabla \nabla_{12} \sqrt{X_{t}}=(1-0.658 L)\left(1-0.623 L^{12}\right) \partial_{t}
\end{aligned}
$$




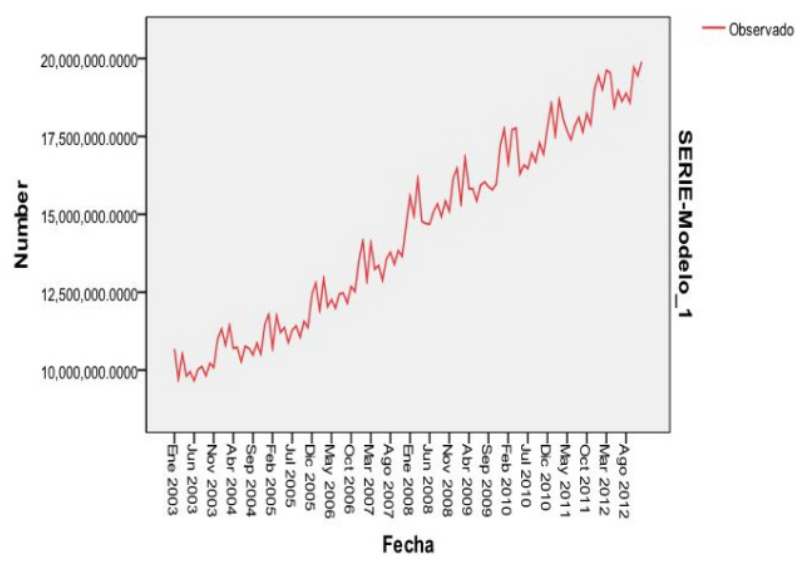

Figura 8. Serie que fue modelada del consumo eléctrico residencial de la Región Lambayeque.

Fuente: Empresa Regional de Servicio Público de Electricidad del Norte S.A.

La figura 8, muestra la serie del consumo eléctrico residencial de la Región Lambayeque, que fue modelada en el periodo 2003-2012, por el modelo ARIMA $(0,1,1)(0,1,1)$, cuyos parámetros que fueron estimados tienen representación estadística a excepción de la constante del modelo, como se demuestra en la tabla Vy tabla VI.

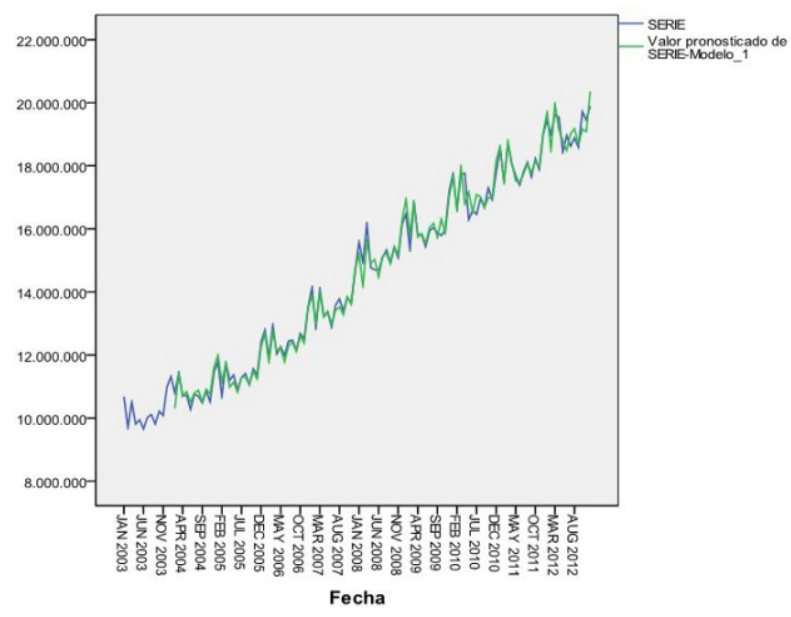

Figura 9. Serie del consumo eléctrico residencial de la región Lambayeque(2003-2012) VS serie que fue modelada del consumo eléctrico residencial de la Región Lambayeque (2003-2012).

La figura 9, muestra la serie en el periodo 20032012, representada por el color azul y la serie que fue modelada en el periodo 2003-2012, representada por el color verde.

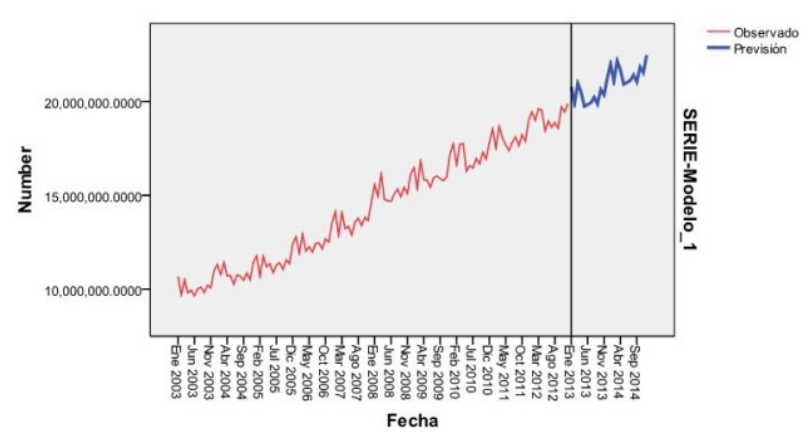

Figura 10. Serie modelada del consumo eléctrico residencial de la Región Lambayeque en el periodo 2003-2012 y para el periodo 2013.2014.

Fuente: Empresa Regional de Servicio Público de Electricidad del Norte S.A.

La figura 10, muestra la serie modelada en el periodo 2003-2012(color rojo) y la serie proyectada en el periodo 2013-2014(color azul); que fue el objetivo del trabajo de investigación.

Tabla 7. Error promedio porcentual de validación que fue calculado.

\begin{tabular}{|c|c|c|c|c|}
\hline SERIE & FIT-3 & ERROR & V. ABS. ERROR & \% ERROR \\
\hline $19,448,890.3349$ & $19,697,603,2767$ & $-248,712.9418$ & 248712.9418 & 1.2788 \\
\hline $19,000,721.6049$ & $18,551,346.6240$ & $449,374.9809$ & 449374.9809 & 2.3650 \\
\hline $19,623,170.6098$ & $19,859,769.8789$ & $-236,599.2691$ & 236599.2691 & 1.2057 \\
\hline $19,540,325.4997$ & $19,074,265.8847$ & $466,059.6150$ & 466059.615 & 2.3851 \\
\hline $18,433,095.8350$ & $18,722,422.4950$ & $-289,326.6600$ & 289326.66 & 1.5696 \\
\hline $18,964,011.7171$ & $18,490,289.3984$ & $473,722.3187$ & 473722.3187 & 2.4980 \\
\hline $18,622,126.0904$ & $18,905,023.6773$ & $-282,897.5869$ & 282897.5869 & 1.5191 \\
\hline $18,885,437.7650$ & $19,160,100.0876$ & $-274,662.3226$ & 274662.3226 & 1.4544 \\
\hline $18,580,114.6360$ & $18,779,747.1468$ & $-199,632.5108$ & 199632.5108 & 1.0744 \\
\hline $19,721,016.7818$ & $19,281,314,8497$ & $439,701.9321$ & 439701.9321 & 22296 \\
\hline $19,447,610.5017$ & $19,024,729.9396$ & $422,880.5621$ & 422880.5621 & 2.1745 \\
\hline $19,894,565.6224$ & $20,197,870.9242$ & $-303,305.3018$ & 303305.3018 & 1.5246 \\
\hline \multicolumn{4}{|c|}{ ERROR PROMEDIO PORCENTUAL DE VALIDACION } & 1.7732 \\
\hline
\end{tabular}

La columna(SERIE), representa la serie de consumo eléctrico residencial de la Región Lambayeque. La columna (FIT-3), representa la serie modelada del consumo eléctrico residencial de la región Lambayeque. La columna (ERROR), representa el error de las series. La columna (V.ABS.ERROR), representa el error absoluto de las series y la columna (\%ERROR), representa el error porcentual de validación de las series.

Fue considerada la serie del consumo eléctrico residencial de la región Lambayeque en el periodo 2003 al 2011. Mediante el modelo 
Arima calculado se modelo la serie del consumo eléctrico residencial de la región Lambayeque en el periodo 2003 al 2012. Finalmente con la serie existente y con la serie modelada se determinó que el error promedio de validación fue 1.7\%.

Tabla 8. Error promedio porcentual histórico que fue calculado.

\begin{tabular}{|c|c|c|c|c|}
\hline$\{9 \mathrm{wl9}$ & FIT-3 & ERROR & V. ABS. ERROR & \% ERROR \\
\hline \multirow{2}{*}{\multicolumn{5}{|c|}{$\begin{array}{l}10,681,452.3990 \\
9694,520.2540\end{array}$}} \\
\hline & & & & \\
\hline \multicolumn{5}{|l|}{$10,512,011.6435$} \\
\hline \multicolumn{5}{|l|}{$9,809,055.0815$} \\
\hline \multicolumn{5}{|l|}{$9,944,721.8198$} \\
\hline \multicolumn{5}{|l|}{$9,657,990.3814$} \\
\hline \multicolumn{5}{|l|}{$10,020,693.4345$} \\
\hline \multicolumn{5}{|l|}{$10,111,910.4885$} \\
\hline \multicolumn{5}{|l|}{$\begin{array}{l}9,814,773.7230 \\
\end{array}$} \\
\hline \multicolumn{5}{|l|}{$10,217,393.3725$} \\
\hline \multicolumn{5}{|l|}{$10,080,175.7964$} \\
\hline $10,995,066.4272$ & & & & \\
\hline $11,321,285.0699$ & & & & \\
\hline $10,794,368.6065$ & $10,306,712.5133$ & $487,656.0932$ & 487656.0932 & 4.5177 \\
\hline $11,437,018.5978$ & $11,422,507.0071$ & $14,511.5907$ & 14511.5907 & 0.1269 \\
\hline $10,700,298.9737$ & $10,695,722.4300$ & $4,576.5437$ & 4576.5437 & 0.0428 \\
\hline $10,729,028.5327$ & $10,839,634.6715$ & $-110,606.1388$ & 110606.1388 & 1.0309 \\
\hline $10,271,309.4141$ & $10,501,952.3508$ & $-230,642.9367$ & 230642.9367 & 2.2455 \\
\hline $10,765,771.2829$ & $10,798,683.9228$ & $-32,912.6399$ & 32912.6399 & 0.3057 \\
\hline $10,694,034.7560$ & $10,882,507.1467$ & $-188,472.3907$ & 188472.3907 & 1.7624 \\
\hline $10,483,284.2580$ & $10,510,855.3339$ & $-27,571.0759$ & 27571.0759 & 0.2630 \\
\hline $10,869,306.4912$ & $10,918,248.3057$ & $-48,941.8145$ & 48941.8145 & 0.4503 \\
\hline $10,511,159.1025$ & $10,760,282.0331$ & $-249,122.9306$ & 249122.9306 & 2.3701 \\
\hline $11,466,714.0635$ & $11,616,206.1567$ & $-149,492.0932$ & 149492.0932 & 1.3037 \\
\hline $11,782,010.3156$ & $11,991,182.1379$ & $-209,171.8223$ & 209171.8223 & 1.7753 \\
\hline $10,679,099.0389$ & $11,134,860.3565$ & $-455,761.3176$ & 455761.3176 & 4.2678 \\
\hline $11,755,125.2694$ & $11,708,994.9499$ & $46,130.3195$ & 46130.3195 & 0.3924 \\
\hline $11,204,416.0412$ & $10,981,122.2867$ & $223,293.7545$ & 223293.7545 & 1.9929 \\
\hline $11,365,038.2747$ & $11,139,658.1979$ & $225,380.0768$ & 225380.0768 & 1.9831 \\
\hline $10,881,320.9710$ & $10,822,557.8086$ & $58,763.1624$ & 58763.1624 & 0.5400 \\
\hline $11,279,247.8984$ & $11,295,320.8163$ & $-16,072.9179$ & 16072.9179 & 0.1425 \\
\hline $11,415,991.5927$ & $11,293,044.8351$ & $122,946.7576$ & 122946.7576 & 1.0770 \\
\hline $11,053,997.5724$ & $11,074,510.0197$ & $-20,512.4473$ & 20512.4473 & 0.1856 \\
\hline $11,561,649.1675$ & $11,478,237.2502$ & $83,411.9173$ & 83411.9173 & 0.7215 \\
\hline $11,364,174.3949$ & $11,238,345.0804$ & $125,829.3145$ & 125829.3145 & 1.1072 \\
\hline $12,414,680.0168$ & $12,260,744.1454$ & $153,935.8714$ & 153935.8714 & 1.2400 \\
\hline $12,797,340.4095$ & $12,687,987.9011$ & $109,352.5084$ & 109352.5084 & 0.8545 \\
\hline $11,898,715.8695$ & $11,779,014.7893$ & $119,701.0802$ & 119701.0802 & 1.0060 \\
\hline $12,948,379.8855$ & $12,751,415.4363$ & $196,964.4492$ & 196964.4492 & 1.5212 \\
\hline $12,030,563.1439$ & $12,129,643.9322$ & $-99,080.7883$ & 99080.7883 & 0.8236 \\
\hline $12,263,969.6914$ & $12,216,389.9989$ & $47,579.6925$ & 47579.6925 & 0.3880 \\
\hline $11,981,618.3528$ & $11,781,308.1838$ & $200,310.1690$ & 200310.169 & 1.6718 \\
\hline $12,443,388.3449$ & $12,297,390.9463$ & $145,997.3986$ & 145997.3986 & 1.1733 \\
\hline $12,471,591.1907$ & $12,411,781.4885$ & $59,809.7022$ & 59809.7022 & 0.4796 \\
\hline $12,144,360.2182$ & $12,113,436.6021$ & $30,923.6161$ & 30923.6161 & 0.2546 \\
\hline $12,683,055.3512$ & $12,598,325.3589$ & $84,729.9923$ & 84729.9923 & 0.6681 \\
\hline $12,513,162.4970$ & $12,378,910.6620$ & $134,251.8350$ & 134251.835 & 1.0729 \\
\hline $13,528,652.2278$ & \begin{tabular}{|l|l}
$13,482,577.5747$ \\
\end{tabular} & $46,074.6531$ & 46074.6531 & 0.3406 \\
\hline $14,143,238.3851$ & $13,896,309.5756$ & $246,928.8095$ & 246928.8095 & 1.7459 \\
\hline $12,848,162.8086$ & $13,009,351.8559$ & $-161,189.0473$ & 161189.0473 & 1.2546 \\
\hline $14,096,941.1085$ & $13,975,072.4495$ & $121,868.6590$ & 121868.659 & 0.8645 \\
\hline $13,236,836.8183$ & $13,201,544.5180$ & $35,292.3003$ & 35292.3003 & 0.2666 \\
\hline $13,355,399.1665$ & $13,387,249.8779$ & $-31,850.7114$ & 31850.7114 & 0.2385 \\
\hline $12,884,231.1352$ & $12,974,988.3449$ & $-90,757.2097$ & 90757.2097 & 0.7044 \\
\hline $13,576,163.5080$ & $13,416,645.2285$ & $159,518.2795$ & 159518.2795 & 1.1750 \\
\hline $13,779,255.6525$ & \begin{tabular}{|l|l}
$13,523,404.1471$ \\
\end{tabular} & $255,851.5054$ & 255851.5054 & 1.8568 \\
\hline $13,390,770.7625$ & $13,273,745.9059$ & $117,024.8566$ & 117024.8566 & 0.8739 \\
\hline $13,832,923.2331$ & $13,837,366.5921$ & $-4,443.3590$ & 4443.359 & 0.0321 \\
\hline $13,650,014.4810$ & $13,608,935.3950$ & $41,079.0860$ & 41079.086 & 0.3009 \\
\hline $14,647,848.6726$ & $14,710,600.1450$ & $-62,751.4724$ & 62751.4724 & 0.4284 \\
\hline $15,592,246.8928$ & $15,192,277.6372$ & $399,969.2556$ & 399969.2556 & 2.5652 \\
\hline $14,936,034.8212$ & $14,182,361.6117$ & $753,673.2095$ & 753673.2095 & 5.0460 \\
\hline $16,165,754.4810$ & $15,610,392.9708$ & $555,361.5102$ & 555361.5102 & 3.4354 \\
\hline $14,771,069.7022$ & \begin{tabular}{|l|l}
$14,915,822.7308$ \\
\end{tabular} & $-144,753.0286$ & 144753.0286 & 0.9800 \\
\hline $14,701,386.3842$ & $15,027,938.0312$ & $-326,551.6470$ & 326551.647 & 2.2212 \\
\hline $14,681,855.8513$ & $14,463,432.9627$ & $218,422.8886$ & 218422.8886 & 1.4877 \\
\hline $15,086,789.0407$ & $15,128,628.6012$ & $-41,839.5605$ & 41839.5605 & 0.2773 \\
\hline $15,331,041.8445$ & $15,231,029.8956$ & $100,011.9489$ & 100011.9489 & 0.6523 \\
\hline $14,927,852.8261$ & $14,888,332.2592$ & $39,520.5669$ & 39520.5669 & 0.2647 \\
\hline $15,432,478.4926$ & $15,421,940.0749$ & $10,538.4177$ & 10538.4177 & 0.0683 \\
\hline $15,089,893.2494$ & $15,203,930.3842$ & $-114,037.1348$ & 114037.1348 & 0.7557 \\
\hline $16,154,286.0492$ & $16,274,543.5285$ & $-120,257.4793$ & 120257.4793 & 0.7444 \\
\hline
\end{tabular}

\begin{tabular}{|c|c|c|c|c|}
\hline & $16,941,864.9562$ & $-467,689.7838$ & 467689.7838 & 2.8389 \\
\hline $15,333,337.1783$ & $15,776,284.1546$ & $-442,946.9763$ & 442946.9763 & 2.8888 \\
\hline $16,839,474.4146$ & $16,856,907.9421$ & $-17,433.5275$ & 17433.5275 & 0.1035 \\
\hline $15,825,837.1336$ & $15,742,660.4525$ & $83,176.6811$ & 83176.6811 & 0.5256 \\
\hline $\begin{array}{l}15,810,960.5694 \\
\end{array}$ & $15,846,830.7589$ & $-35,870.1895$ & 35870.1895 & 0.2269 \\
\hline $15,431,242.1667$ & $15,537,209.6351$ & $-105,967.4684$ & 105967.4684 & 0.6867 \\
\hline $15,939,833.0872$ & $16,037,076.3038$ & $-97,243.2166$ & 97243.2166 & 0.6101 \\
\hline $16,036,997.0166$ & $16,173,946.2563$ & $-136,949.2397$ & 136949.2397 & 0.8540 \\
\hline $15,883,547.4394$ & $\begin{array}{l}15,729,872.1108 \\
\end{array}$ & $153,675.3286$ & 153675.3286 & 0.9675 \\
\hline $\begin{array}{l}15,787,552.0174 \\
\end{array}$ & $16,311,325.6024$ & $-523,773.5850$ & 523773.585 & 3.3176 \\
\hline $15,977,840.0258$ & $15,857,832.6786$ & $120,007.3472$ & 120007.3472 & 0.7511 \\
\hline $17,199,142.6215$ & $17,018,013.0239$ & $181,129.5976$ & 181129.5976 & 1.0531 \\
\hline $17,746,035.5723$ & $17,654,763.9182$ & $91,271.6541$ & 91271.6541 & 0.5143 \\
\hline $16,608,584.0489$ & $16,597,264.5471$ & $11,319.5018$ & 11319.5018 & 0.0682 \\
\hline $17,717,674.2299$ & $17,982,899.0183$ & $-265,224.7884$ & 265224.7884 & 1.4970 \\
\hline $17,767,110.5620$ & $16,786,333.9178$ & $980,776.6442$ & 980776.6442 & 5.5202 \\
\hline $16,291,034.4371$ & $17,161,689.5714$ & $-870,655.1343$ & 870655.1343 & 5.3444 \\
\hline $16,581,531.6673$ & $16,522,601.8346$ & $58,929.8327$ & 58929.8327 & 0.3554 \\
\hline $16,461,896.2115$ & $17,086,239.9698$ & $-624,343.7583$ & 624343.7583 & 3.7927 \\
\hline $16,968,356.0486$ & $17,018,144.4673$ & $-49,788.4187$ & 49788.4187 & 0.2934 \\
\hline $16,675,570.7629$ & $16,687,626.1636$ & $-12,055.4007$ & 12055.4007 & 0.0723 \\
\hline $17,297,082.2780$ & $16,983,195.6430$ & $313,886.6350$ & 313886.635 & 1.8147 \\
\hline $16,926,610.3006$ & $16,989,174.8795$ & $-62,564.5789$ & 62564.5789 & 0.3696 \\
\hline $17,800,442.6170$ & $18,162,158.8948$ & $-361,716.2778$ & 361716.2778 & 2.0321 \\
\hline $18,556,209.4443$ & $18,617,352.2841$ & $-61,142.8398$ & 61142.8398 & 0.3295 \\
\hline $17,502,862.1410$ & $17,461,642.7196$ & $41,219.4214$ & 41219.4214 & 0.2355 \\
\hline $18,695,173.1224$ & $18,787,028.0806$ & $-91,854.9582$ & 91854.9582 & 0.4913 \\
\hline $18,056,008.1570$ & $18,065,229.7566$ & $-9,221.5996$ & 9221.5996 & 0.0511 \\
\hline $17,675,478.9929$ & $17,520,346.4555$ & $155,132.5374$ & 155132.5374 & 0.8777 \\
\hline $17,386,876.8032$ & $17,470,274.3772$ & $-83,397.5740$ & 83397.574 & 0.4797 \\
\hline $17,833,461.2241$ & $17,741,500.5736$ & $91,960.6505$ & 91960.6505 & 0.5157 \\
\hline $18,113,930.2470$ & $18,064,713.9747$ & $49,216.2723$ & 49216.2723 & 0.2717 \\
\hline $17,643,722.6401$ & $17,766,257.3430$ & $-122,534.7029$ & 122534.7029 & 0.6945 \\
\hline $18,230,715.0877$ & $18,158,134.7815$ & $72,580.3062$ & 72580.3062 & 0.3981 \\
\hline $17,878,734.1632$ & $17,974,631.6762$ & $-95,897.5130$ & 95897.513 & 0.5364 \\
\hline $19,011,152.2989$ & $19,045,377.6051$ & $-34,225.3062$ & 34225.3062 & 0.1800 \\
\hline $19,448,890.3349$ & $19,698,885.7554$ & $-249,995.4205$ & 249995.4205 & 1.2854 \\
\hline $19,000,721.6049$ & $18,478,612.2577$ & $522,109.3472$ & 522109.3472 & 2.7478 \\
\hline $19,623,170.6098$ & $19,963,713.2288$ & $-340,542.6190$ & 340542.619 & 1.7354 \\
\hline $19,540,325.4997$ & $19,156,250.1921$ & $384,075.3076$ & 384075.3076 & 1.9656 \\
\hline $18,433,095.8350$ & $18,789,965.8247$ & $-356,869.9897$ & 356869.9897 & 1.9360 \\
\hline $18,964,011.7171$ & $18,489,700.2911$ & $474,311.4260$ & 474311.426 & 2.5011 \\
\hline $18,622,126.0904$ & $19,018,561.3927$ & $-396,435.3023$ & 396435.3023 & 2.1288 \\
\hline $18,885,437.7650$ & $19,178,932.6516$ & $-293,494.8866$ & 293494.8866 & 1.5541 \\
\hline $18,580,114.6360$ & $18,694,725.6954$ & $-114,611.0594$ & 114611.0594 & 0.6168 \\
\hline $19,721,016.7818$ & $19,160,300.5968$ & $560,716.1850$ & 560716.185 & 2.8432 \\
\hline $19,447,610.5017$ & $19,080,436.0703$ & $367,174.4314$ & 367174.4314 & 1.8880 \\
\hline $19,894,565.6224$ & $20,358,811.2101$ & $-464,245.5877$ & 464245.5877 & 2.3335 \\
\hline & $20,796,496.1037$ & & & \\
\hline & $19,891,878.5073$ & & & \\
\hline & $20,971,142.2813$ & & & \\
\hline & $20,495,078.5979$ & & & \\
\hline & $19,746,962.9693$ & & & \\
\hline & $19,838,260.2119$ & & & \\
\hline & $19,940,902.2567$ & & & \\
\hline & $20,233,165.3844$ & & & \\
\hline & $19,867,686.2080$ & & & \\
\hline & $20,635,245.6153$ & & & \\
\hline & $20,355,631.0994$ & & & \\
\hline & $21,265,870.5776$ & & & \\
\hline & $21,998,339.4222$ & & & \\
\hline & $21,067,815.8580$ & & & \\
\hline & $22,178,166.2742$ & & & \\
\hline & $21,688,651.7766$ & & & \\
\hline & $20,919,012.4625$ & & & \\
\hline & $21,013,083.2571$ & & & \\
\hline & $21,118,822.3595$ & & & \\
\hline & $21,419,656.1575$ & & & \\
\hline & $21,043,700.6518$ & & & \\
\hline & $21,833,498.0173$ & & & \\
\hline & $21,545,983.1297$ & & & \\
\hline \multirow{2}{*}{\multicolumn{4}{|c|}{\begin{tabular}{l|l}
$22,482,219.1577$ & \\
ERROR PROMEDIO PORCENTUAL
\end{tabular}}} & \\
\hline & & & & 1.2470 \\
\hline
\end{tabular}


La columna (SERIE), representa la serie de consumo eléctrico residencial de la Región Lambayeque. La columna (FIT-3), representa la serie modelada del consumo eléctrico residencial de la región Lambayeque. La columna (ERROR), representa el error de las series. La columna (V.ABS.ERROR), represento el error absoluto de las series y la columna (\%ERROR), represento el error promedio porcentual histórico de las series.

Fue considerada la serie del consumo eléctrico residencial de la región Lambayeque en el periodo 2003 al 2012. Mediante el modelo Arima calculado se modelo la serie del consumo eléctrico residencial de la región Lambayeque en el periodo 2003 al 2014. Finalmente con la serie existente y con la serie modelada se determinó que el error promedio porcentual histórico fue $1.2 \%$.

\section{DISCUCION}

El valor de $\lambda$, es proporcionado en forma automática por el programa SPPSS y se aproxima a 0,5 y no al cero, que significa según la familia de transformaciones de Box Cox; que a la serie se le transformo aplicando raíz cuadrada, consiguiendo de esta manera una serie estacionaria en varianza.

En este trabajo, se utilizó la serie que se aplicó en forma simultánea una diferencia regular y una diferencia estacional, porque el valor de la media fue la menor, lo que significó que es la serie que tiene mayor estabilidad en la media así como también la desviación típica fue la menor. Se puede decir que se tiene una serie estacionaria en media y en estacionalidad.

A esta serie estacionaria en varianza, media y estacionalidad, se le aplico 11 retardos para obtener su autocorrelación simple y parcial muestral, luego estas autocorrelaciones muéstrales fueron analizadas con las autocorrelaciones simple y parcial teóricas para determinar las órdenes de los procesos autorregresivos y de medias móviles (p,q) de la estructura regular.
Para la estructura estacional a esta misma serie estacionaria en varianza, media y estacionalidad, se le aplico 60 retardos y se logró obtener su autocorrelación simple y parcial muestral, luego se analizaron con las autocorrelaciones simple y muestral teóricas y se determinó las órdenes de los procesos autorregresivos y de medias móviles $(\mathrm{P}, \mathrm{Q})$. Finalmente se logró obtener el modelo $\operatorname{ARIMA}(0,1,1)(0,1,1)$.

Los parámetros o coeficientes $\left(\begin{array}{lll}\theta_{1} & y & \Theta_{1}\end{array}\right)$ que fueron estimados, con el modelo ARIMA $(0,1,1)(0,1,1)$ calculado, tienen representación estadística, a excepción de la constante del modelo.

El error promedio porcentual histórico fue de $1.2 \mathrm{y}$ el error promedio porcentual de validación fue de 1.7 .

Por lo tanto el modelo ARIMA $(0,1,1)(0,1,1)$ calculado, es el modelo que mejor se ajustó a la serie "consumos de energía eléctrica residencial de la región Lambayeque". Este modelo matemáticamente se escribió como:

$X_{t}-X_{t-1}-X_{t-12}+X_{t-13}=\partial_{t}-\theta_{1} \partial_{t-1}-\Theta_{1} \partial_{t-12}+\theta_{1} \Theta_{1} \partial_{t-13} \quad$ Ec. 5

Se demuestra, que el modelo ARIMA $(0,1,1)(0,1,1)$ que fue calculado es correcto, ya que se ajusta a la serie estudiada. Por lo tanto se puede afirmar que en la región de Lambayeque fue posible modelar los consumos de energía eléctrica residencial, utilizando la metodología de Box y Jenkins o metodología ARIMA, esto debido a que la serie estudiada se puede convertir en estacionaria, lográndose de esta manera una alta probabilidad de éxito en la modelación, a través de dicha metodología.

La metodología Arima permite modelar los consumos de energía eléctrica en el corto plazo, así lo demuestran los trabajos realizados por Patricia Carbajal Olaya (2003), quien modela los consumos de energía eléctrica en los municipios de Colombia, utilizando la metodología propuesta por Box JenKis o Arima; por Francisco Reyes Lora (2016), quien estima el comportamiento de la curva de demanda de energía eléctrica, mediante modelos Arima o 
Redes Neuronales; y Manuel Chong y Renato Aguilar (2016), quienes concluyen que la metodología Arima es mejor para la proyección del consumo de energía eléctrica para clientes residenciales en Ecuador. La metodología Arima también permite modelar con éxito el consumo de energía eléctrica en el mediano plazo, así se demuestra con el presente trabajo de investigación.

El presente trabajo de investigación, indico el error promedio histórico y el error promedio porcentual de validación, valores muy por debajo del $5 \%$ permitido por dicha metodología. Cabe indicar que este error promedio, no fue presentado por los autores mencionados líneas arriba.

\section{CONCLUCIONES}

1. El modelo ARIMA obtenido fue $(0,1,1)(0,1,1)$, y matemáticamente se escribió como:

$X_{t}-X_{t-1}-X_{t-12}+X_{t-13}=\partial_{t}-\theta_{1} \partial_{t-1}-\Theta_{1} \partial_{t-12}+\theta_{1} \Theta_{1} \partial_{t-13}$

Ec. 5

2. La metodología ARIMA permitió modelar el consumo de energía eléctrica residencial en la región Lambayeque.

En este trabajo, se estabilizo la varianza aplicando raíz cuadrada, luego se estabilizo la media al diferenciar su estructura regular, finalmente se estabilizo la estacionalidad al diferenciar su estructura estacional.

En la serie estacionaria en varianza, media y estacionalidad se logró obtener su autocorrelación simple y parcial muestral, determinándose las órdenes de los procesos autorregresivos y de medias móviles $(p, q)$ de la estructura regular. En la serie estacionaria en varianza, media y estacionalidad se logró obtener su autocorrelación simple y parcial muestral, determinándose las órdenes de los procesos autorregresivos y de medias móviles $(\mathrm{P}, \mathrm{Q})$ de la estructura estacional. Finalmente se obtiene el modelo ARIMA.

Los parámetros o coeficientes $(11 y \theta \Theta)$, tienen representación estadística, a excepción de la constante del modelo.

El error promedio porcentual histórico fue de 1.2 y el error promedio porcentual de validación fue de 1.7 .

\section{BI BLIOGRAFÍA}

González Chávez, S. (2007). Modelos energéticos metodologías, cálculos y aplicaciones. Lima. Perú.

Gutiérrez Moya, E. (2003). Tesis sobre La Demanda Residencial de Energía Eléctrica en la Comunidad Autónoma de Andalucía. Un Análisis cuantitativo. Sevilla, España: recuperado de www.cervantesvirtual.com/.../1ademanda-residencial-de-energíaelectric.

Gujarati, D. (1997). Econometría (3º ed.). Santafé de Bogotá, Colombia: McGrawHill.

Universidad Pontificia Comillas-Madrid. Metodología ARIMA aplicada a análisis y predicción de series. España: en la dirección web http://www.utp.edu.com

Universidad autónoma de Madrid (2004). Curso de predicción económica y empresarial. Modelo ARIMA. España: recuperada de: http://www.uam.es/predysim.

Bowerman, B., O'Connell, R. y Koehler, A. (2006). Pronóstico, series de tiempo y regresión. Un enfoque aplicativo ( $4^{\circ} \mathrm{ed}$.). México: CENGAGE learning.

Fernandez, Adrian. Análisis de series de tiempo: e $\mathrm{n} \quad \mathrm{a}$ d i r e c c i ó $\mathrm{n}$ w e b http://www.ccee.edu.uy

Hernández Sampieri, R., Fernández, C. y Baptista, P. (2006). Metodología de la investigation $\left(4^{\circ}\right.$ ed.). México: McGraw-Hill.

Peña Sánchez de Rivera D. (1989). Estadística modelos y métodos. 2. Modelos lineales y series temporales ( $2^{\circ}$ ed.). Madrid, España: Alianza Universidad Textos. 
Patricia Carbajal Olaya (2003). Articulo estudio del pronóstico de la demanda de energía eléctrica, utilizando modelos de series de tiempo. Colombia.

Francisco Reyes Lora (2016), Tesis predicción de la demanda eléctrica: comparativa Arima - redes neuronales mediante Software SPSS. España.

Manuel Chong y Renato Aguilar (2016). Artículo proyección de serie de tiempo para el consumo de energía eléctrica a clientes residenciales en Ecuador. Ecuador.

\section{CORRESPONDENCIA}

Walter Cabrera Torres

Húsares de Junin $N^{\circ} 146$ - José Leonardo

Ortiz - Chiclayo

linder_ct@hotm $\lambda \underline{\text { il.com }}$ 